\title{
IUFOST2006/1091 Sensory Cues for Emotional Responses to Foods \& Drinks
}

\author{
D. Thomson \\ MMR Research Worldwide, Wallingford House, 46 High Street, Wallingford, OX10 0DB Oxford, United \\ Kingdom \\ d.thomson@mmr-research.com
}

The very existence of the fragrance industry and the fact that it has flourished for millennia, testifies to the fact that the human senses provide a very direct conduit to our emotions. If further evidence were required, then simply check out the imagery in any perfumery in any department store or the advertising messages communicated by perfumers on screen or in print.

When the sensory characteristics of a product cue an emotional response, it is often referred to as a SensoEmotional relationship and these sensory characteristics are said to be emotionally active.

SensoEmotional relationships are far more powerful and far more widespread than most of us imagine. The linkages between sweetness and feelings of safety, comfort and love, are very well known, as is the relationship between the melt-in-the-mouth characteristics of chocolate and sensuousness. It's perhaps less obvious that a seemingly ordinary product such as dried pasta, for example, could be SensoEmotionally active. However, the sensory differences in shape and texture between tagliatelle and spaghetti undoubtedly cue measurably different emotional reactions, as does the colour difference between plain tagliatelle and green tagliatelle.

SensoEmotional relationships are bringing a powerful new dimension to branding. Marketers now recognise that by featuring emotionally active sensory characteristics in brand architecture and brand communication, it's possible to cue emotions that are fundamental to the way they want consumers to perceive their brands. For example, richness, melt-in-the-mouth and smoothness are widely used to reinforce the notion of sensuousness and feelings of indulgence in chocolate branding.

This presentation will describe how relationships are formed between the sensory characteristics of products and the emotional characteristics of brands, and how these are used in branding. In doing so, it will also allude to the processes consumers use to structure information (e.g. Means End Chain Theory and the Theory of Personal Constructs) and the research procedures used to access these knowledge structures (e.g. Method of Diads/Triads and Laddering).

Finally, a practical process for identifying SensoEmotional linkages will be described. 